\title{
CONTRACT TITLE AND NUMBER:
}

SOXAL Combined SOx/NOx Flue Gas Control Demonstration

DE AC 22-91 PC 91347

Contractor Name:

AQUATECH Systems

7 Powder Horn Drive

Warren, NJ 07059-5191

Contract Period: $\quad 9-10-91-7-1-93$

1. Contract Objective: No Change

Summary of Work -

AQUATECH Systems, a business unit of Allied-Signal Inc., proposes to demonstrate the technical viability and cost effectiveness of the SOXAL process a combined $\mathrm{SO}_{X} \mathrm{NO}_{X}$ control process on a $3 \mathrm{MW}$ equivalent flue gas slip stream from Niagara Mohawk Power Corporation, Dunkirk Steam Station Boiler \#4, a coal fired boiler. The SOXAL process combines $90+\%$ sulfur dioxide removal from the flue gas using a sodium based scrubbing solution and regeneration of the spent scrubbing liquor using AQUATECH Systems' proprietary bipolar membrane technology. This regeneration step recovers a stream of sulfur dioxide suitable for subsequent processing to salable sulfur or sulfuric acid.

Additionally $90+\%$ control of $\mathrm{NO}_{x}$ gases can be achieved in combination with conventional urea/methanol injection of $\mathrm{NO}_{2}$ gas into the duct. The SOXAL process is applicable to both utility and industrial scale boilers using either high or low sulfur coal.

The SOXAL demonstration Program began September 10, 1991 and is approximately 22 months in duration. 
During the 6 months of scheduled operations period, begun in January 1993, data will be collected from the SOXAL system to define:

1) $\mathrm{SO}_{2}$ and $\mathrm{NO}_{x}$ control efficiencies

2) Current efficiency for the regeneration unit.

3) Sulfate oxidation in the absorber.

4) Make-up reagent rates.

5) Product quality including concentrations and compositions.

6) System integration and control philosophy

7) Membrane stability and performance with respect to foulants.

The program is expected to be concluded by November 1993.

Technical Approach Changes: No Change

Contract (by reporting element):

Task 1: $\quad$ Program Definition - Complete

Task 2: $\quad$ Engineering, Design and Construction - Complete

Task 3: $\quad$ Shakedown, Parametric Testing, and Disassembly

\section{Subtask 3.1: Shakedown}

Process shakedown was completed in December 1992. However, several key items have not yet been satisfactorily commissioned. The Lear-Siegler gas analyzer has not yet operated. The $\mathrm{NO}_{2}$ injection system was checked out and commissioned in June. 


\section{Subtask 3.2: Parametric Tests}

Parametric testing commenced on January 6, 1993. Between January 8 and March 5, the pilot plant was operated continuously ( 7 days per week, 24 hours per day). The plant was easy to operate and control. However, several problems made data collection during this period difficult. These included the lack of any gas analyses, unreliable flow sensors, and inappropriate materials of construction.

Niagara Mohawk shut down Unit No. 4 boiler on March 5 and attempted to resume operations on April 7. During the shutdown, we addressed a number of major and minor problems affecting plant operation. Most notably, we arranged for an outside CEM service (Acres International) to provide analyses of our inlet and outlet gas streams. Additionally, we had engineering companies (United Engineers and Constructors, Radian Corporation) review plant equipment and operations. They have recommended a number of minor instrumentation changes to improve control and reliability of the plant.

We also learned that Niagara Mohawk's summer schedule would not provide base-loaded boiler operation for the remainder of our testing. They planned to shut the boiler down during the weekends and operate it at reduced load during the night. We adapted to this change by rescheduling our testing for 24- hour, 5 day operation. operation of either the absorption or regeneration processes was planned to provide a testing window with consistent flue gas conditions.

Parametric testing resumed on April 26. The first two weeks of testing were used to reestablish baseline operating conditions with continuous operation. Following that we used our new procedures to verify that test results from decoupled operation would be comparable to results from continuous operation. The remaining weeks in May and June were devoted to an assortment of absorption and regeneration tests. These tests included an artificial increase in $\mathrm{SO}_{2}$ concentration and preliminary tests of $\mathrm{NO}_{2}$ injection. The attached table summarizes the data taken during this quarter. A thorough evaluation of these results has not been completed. 
Lear Siegler attempted to fix their gas analysis equipment during April and May, without success. We are attempting to negotiate a return of their equipment and a refund of our money.

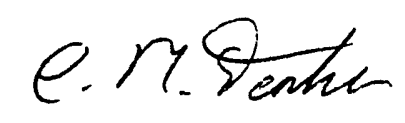

\section{Clifford M. Denker}

Program Manager

\section{$\mathrm{CMD}: \mathrm{cml}$}

\section{Attachments:}

Milestone Schedule - June 1993

Parametric Testing Summary - April thru June 1993 


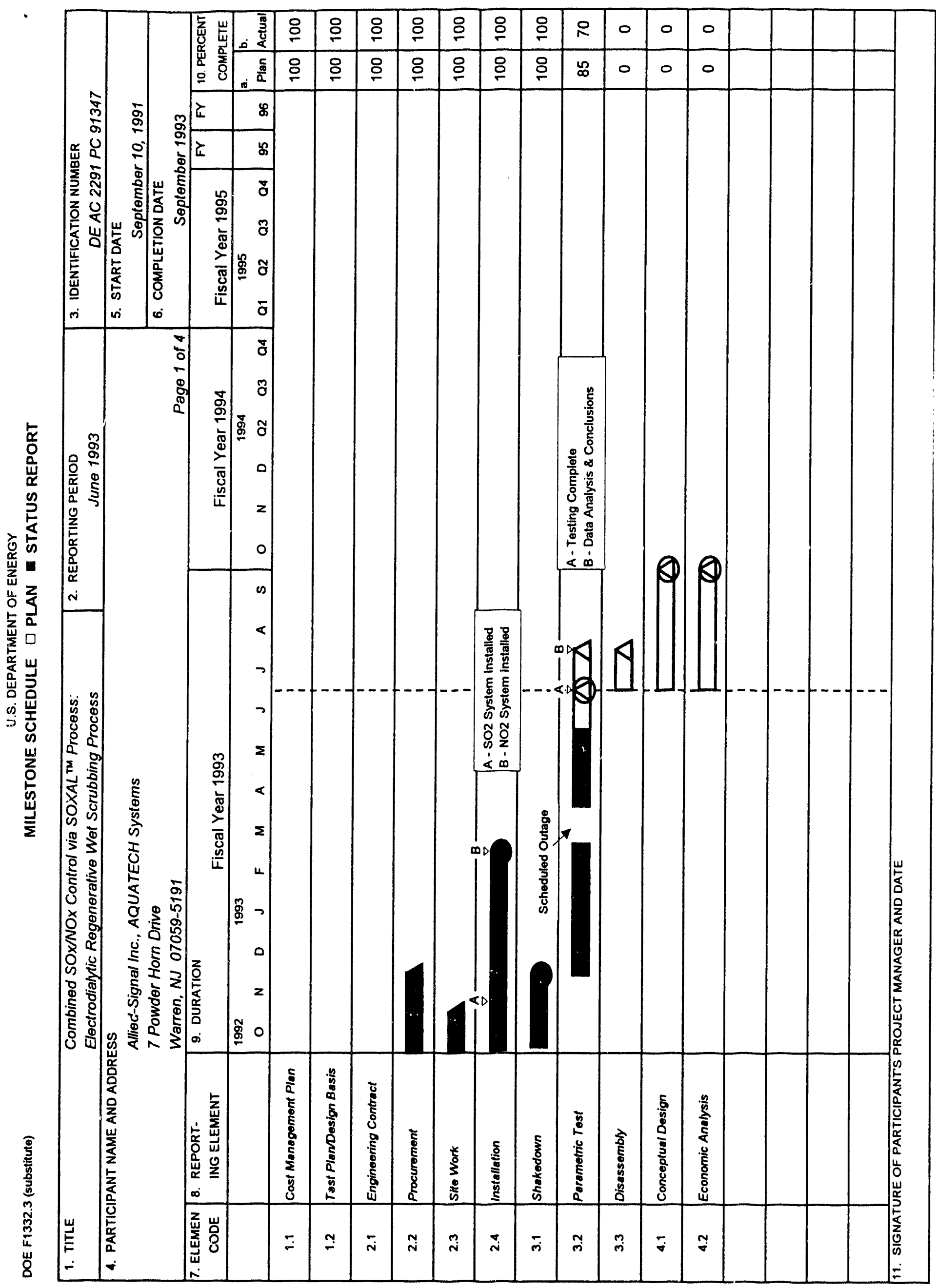


Parametric Testing Summary

April - June 1993

Test Description and Results - Daily Averages'

\begin{tabular}{|c|c|c|c|c|c|c|c|}
\hline $\begin{array}{l}\text { Test } \\
\text { Code }\end{array}$ & $\begin{array}{l}\text { Test } \\
\text { Date }\end{array}$ & Test Description & $\begin{array}{c}\mathrm{SO} 2 \\
\text { Concentration } \\
\text { (ppm) }\end{array}$ & $\begin{array}{l}\text { Flue Gas } \\
\text { Flow Rate } \\
\text { (DSCFM) }\end{array}$ & $\begin{array}{c}\mathrm{SO}_{2} \\
\text { Absorption } \\
(\%)\end{array}$ & $\begin{array}{c}\text { Power } \\
\text { Consumed } \\
\text { (kWh/t SO2) }\end{array}$ & $\begin{array}{c}\text { Current } \\
\text { Efficiency } \\
(\%)\end{array}$ \\
\hline A & $4 / 26$ & Simultaneous ${ }^{2}$ Baseline & & & & & 101.4 \\
\hline A & $4 / 27$ & Simultaneous ${ }^{2}$ Baseline & & & & & 81.6 \\
\hline A & $4 / 28$ & Simultaneous ${ }^{2}$ Baseline & & & & & 83.9 \\
\hline A & $4 / 29$ & Simultaneous ${ }^{2}$ Baseline & 916 & $7992^{3}$ & 98.8 & & 98.2 \\
\hline A & $4 / 30$ & Simultaneous ${ }^{2}$ Baseline & 1,202 & & 99.3 & & 86.6 \\
\hline AH1 & $5 / 6$ & Simultaneous ${ }^{2}$ Baseline with $1.25 \times$ Foed to Base & 1,062 & 5,205 & 96.3 & & 63.1 \\
\hline $\mathrm{A} / \mathrm{H} 1$ & $5 / 7$ & Simultaneous ${ }^{2}$ Baseline with $1.25 \times$ Feed to Base & 1,115 & 4,798 & 97.5 & & 60.8 \\
\hline $\mathrm{A} / \mathrm{H} 2$ & $5 / 3$ & Simultaneous ${ }^{2}$ Baseline with $1.5 \times$ Feed to Base & 1,149 & $7562^{3}$ & 98.9 & & 90.7 \\
\hline $\mathrm{A} / \mathrm{H} 2$ & $5 / 4$ & Simultaneous ${ }^{2}$ Baseline with $1.5 \times$ Feed to Base & 1,189 & $7366^{3}$ & 97.2 & & 71.7 \\
\hline $\mathrm{A} / \mathrm{H} 2$ & $5 / 5$ & Simultaneous ${ }^{2}$ Baseline with $1.5 \times$ Feed to Base & 988 & 4,758 & 96.6 & & 66.0 \\
\hline B & $5 / 10$ & Absorber Baseline & 861 & 5,201 & 96.8 & & \\
\hline B & $5 / 11$ & Absorber Baseline & 1,385 & 5,609 & 98.7 & & \\
\hline B & $5 / 12$ & Absorber Baseline & 1,365 & 5,201 & 98.8 & & \\
\hline B & $5 / 28$ & Absorber Baseline & 1,316 & 5,709 & 98.4 & & \\
\hline C & $5 / 13$ & Cellstack Baseline & & & & 1,122 & 93.3 \\
\hline C & $5 / 14$ & Cellstack Baseline & & & & 1,251 & 90.3 \\
\hline $\mathrm{c}$ & $5 / 26$ & Cellstack Baseline & & & & 1,082 & 96.4 \\
\hline D1 & $5 / 17$ & Absorber Bottoms at 5:1 Ratio Bisulfite:Sulfite & 1,361 & 5,309 & 99.6 & & \\
\hline D1 & $5 / 18$ & Absorber Bottoms at 5:1 Ratio Bisulfite: Sulfite & 1,278 & 5,175 & 98.9 & & \\
\hline D1 & $5 / 19$ & Absorber Bottoms at 5:1 Ratio Bisulfite:Sulfite & 1,085 & 5,711 & 91.9 & & \\
\hline D1 & $6 / 10$ & Absorber Bottoms at 5:1 Ratio Bottoms & 1,182 & 5,402 & 97.7 & & \\
\hline $\mathrm{D} 2$ & $5 / 20$ & Absorber Bottoms at 1:1 Ratio Bisulfite:Sulfite & 1,086 & 5,744 & 100.0 & & \\
\hline E2 & $5 / 21$ & Absorber Recycle at $60 \mathrm{gpm}$ & 1,146 & 5,880 & 97.9 & & \\
\hline E3 & $5 / 24$ & Absorber Recycle at $\mathbf{4 5} \mathrm{gpm}$ & 1,123 & 5.771 & 95.9 & & \\
\hline $\mathrm{F} 1$ & $5 / 25$ & Absorber with One Stage & 1,211 & 5,865 & 82.7 & & \\
\hline$G$ & $6 / 7$ & Absorber with Recycled SO2 & 2,141 & 5,407 & 99.6 & & \\
\hline $\mathbf{G}$ & $6 / 8$ & Absorber with Recycled SO2 & 2,167 & 5,394 & 100.0 & & \\
\hline G2 & $6 / 9$ & Absorber with Recycled SO2, 5:1 Ratio Bottoms & 2,228 & 5,377 & 99.4 & & \\
\hline$H$ & $6 / 30$ & Simultaneous with Recycled $\mathrm{SO} 2$ & 1,977 & 5,046 & 99.9 & & \\
\hline $\mathrm{H} 2$ & $5 / 27$ & Cellstack with $1.25 \times$ Feed to Base & & & & 1,131 & 79.0 \\
\hline 11 & $6 / 1$ & Cellstack at $80 \mathrm{ASF}$ & & & & 1,049 & 101.2 \\
\hline 12 & $6 / 2$ & Cellstack at 125 ASF & & & & 1,331 & 77.8 \\
\hline J1 & $6 / 3$ & Cellstack at $80 \%$ Acid Conversion & & & & 1,334 & 82.0 \\
\hline$\sqrt{ } 2$ & $6 / 4$ & Cellstack at $120 \%$ Acid Conversion & & & & 1,292 & 81.4 \\
\hline K & $6 / 24$ & NO2 injection at $150 \mathrm{ppm}$ & 817 & 5,118 & 99.9 & & \\
\hline $\mathrm{K} 2$ & $6 / 28$ & NO2 Injection at $60 \mathrm{ppm}$ & 1,208 & 5,117 & 99.2 & & \\
\hline $\mathrm{K} 2$ & $6 / 29$ & NO2 Injection at $70 \mathrm{ppm}$ & 1,118 & 4,869 & 99.9 & & \\
\hline U1 & $6 / 14$ & Absorber Recycle at $45 \mathrm{gpm}, 5: 1$ Ratio Bottoms & 1,069 & 5,398 & 93.6 & & \\
\hline U2 & $6 / 15$ & Absorber Recycle at 75 gpm, 5:1 Ratio Bottoms & 1,097 & 5,376 & 94.3 & & \\
\hline V & $6 / 16$ & Cellstack with 5:1 Ratio Feed & & & & 1,152 & 66.6 \\
\hline
\end{tabular}

Notes:

1 Data taken between $4 / 26 / 93-6 / 30 / 93$. Each data point typically represents the average of four measurements taken during an eight-hour test.

2 Simultaneous indicates continuous operation of both absorption and regeneration processes. All other tests conducted are decoupled.

3 These flow rates in ACFM. All others in Dry SCFM. 
Parametric Testing Summary

April - June 1993

SO2 Inlet Concentration

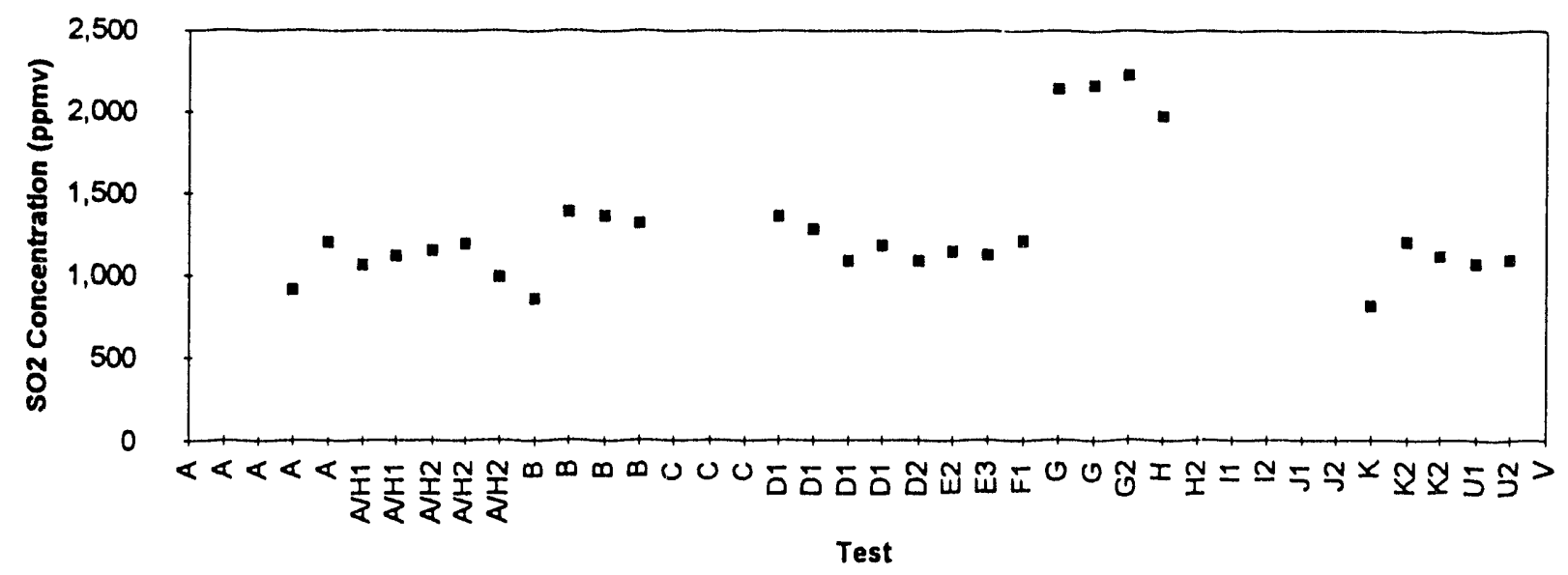

Flue Gas Flow Rate

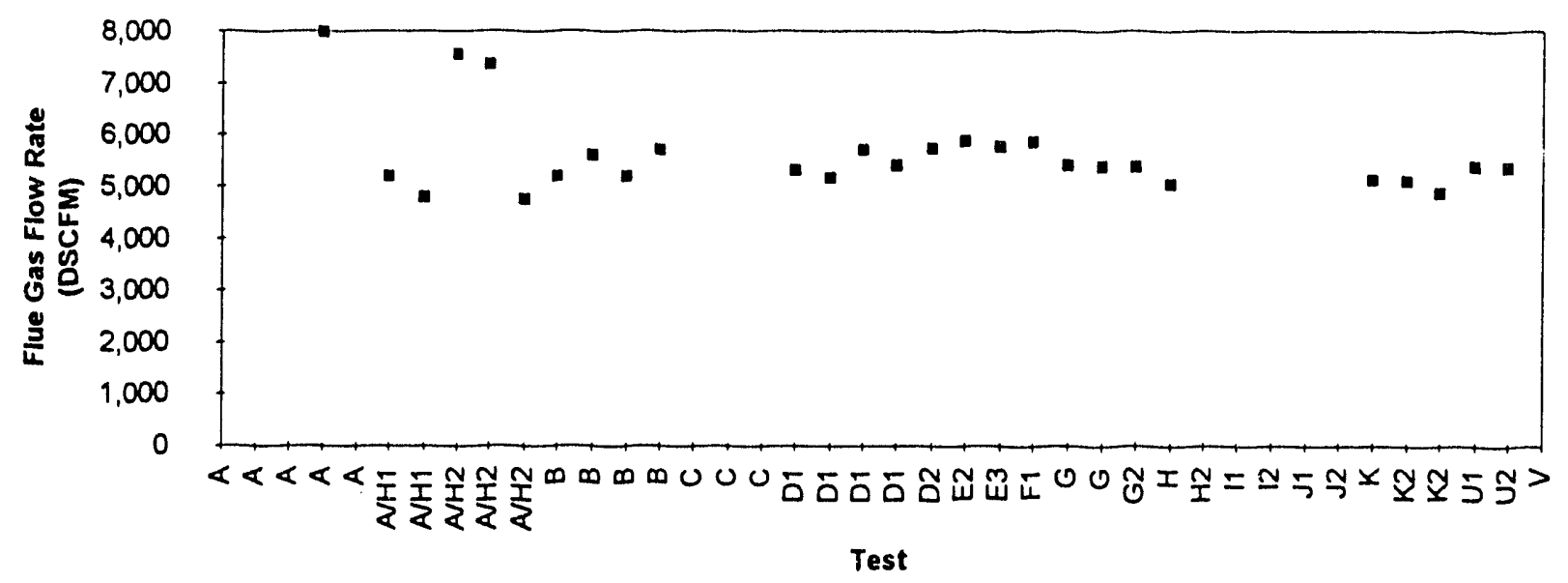

SO2 Absorption Efficiency

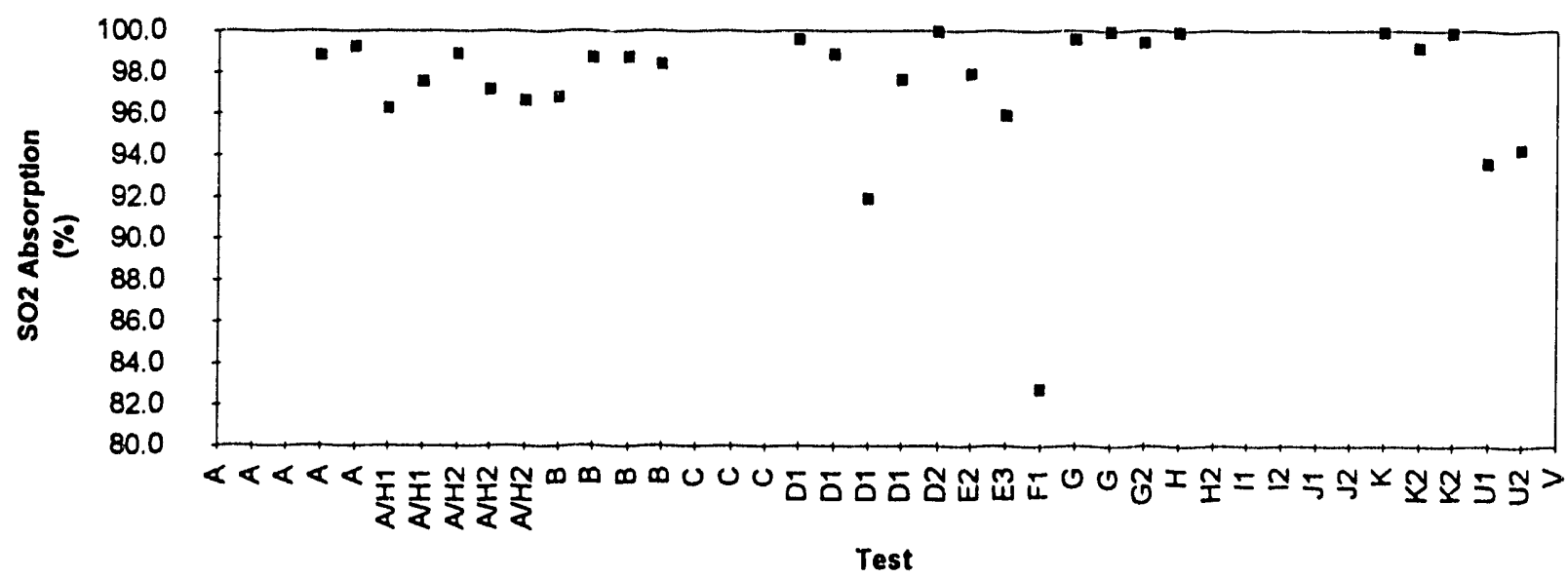




\section{Cell Stack Power Consumption}

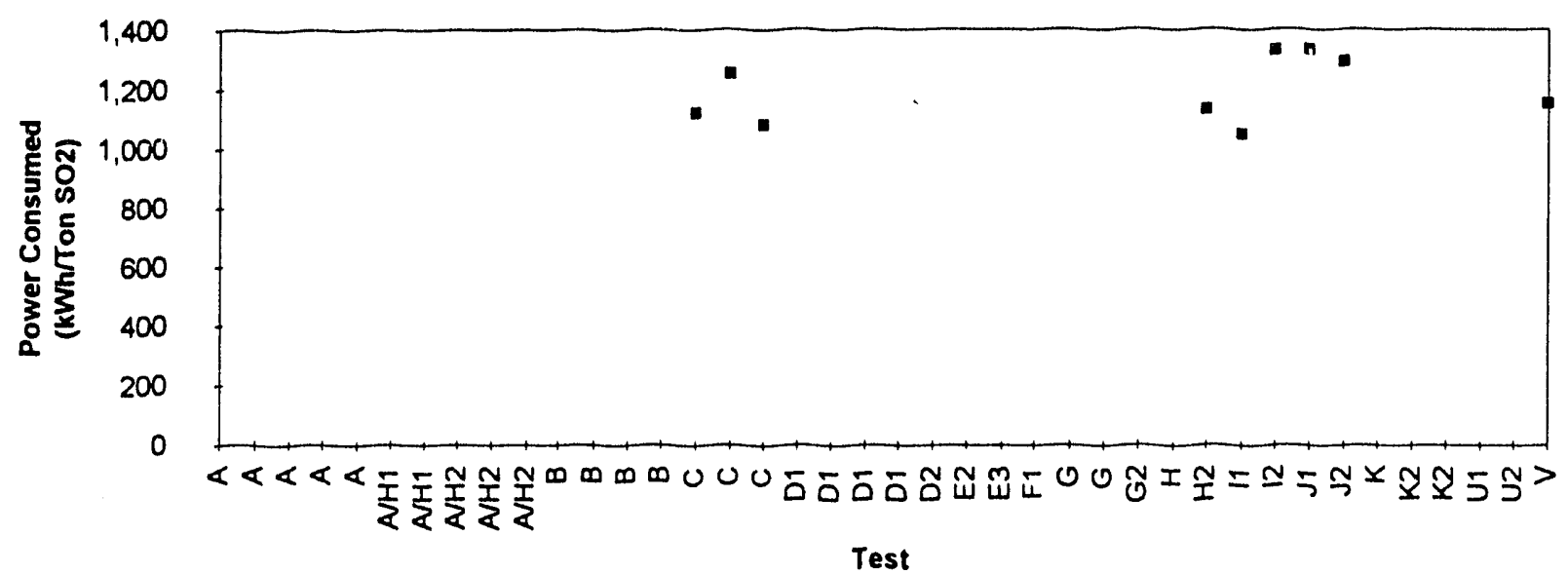

Cell Stack Current Efficiency

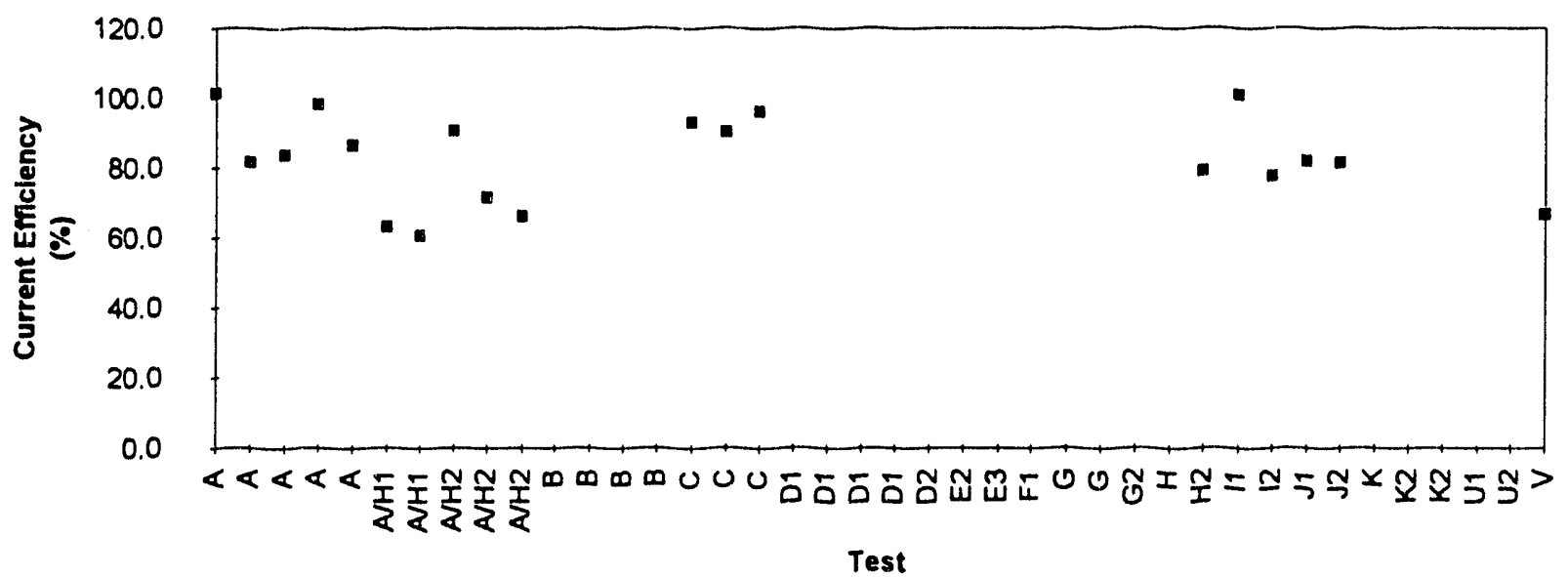


